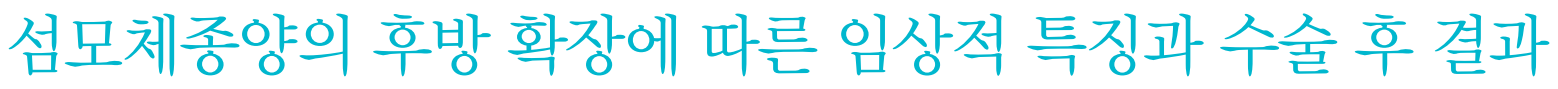

\title{
Effects of Posterior Extension on the Clinical Characteristics and Surgical Outcomes of Ciliary Body Tumors
}

\author{
김수한 ${ }^{1}$, 이승규 ${ }^{2}$, 이지환 ${ }^{2}$, 이성철 $^{2}$ \\ Soo-Han Kim , Christopher Seungkyu Lee², Jihwan Lee², Sung Chul Lee² \\ ${ }^{1}$ 연세대학교 원주의과대학 안과학교실, ${ }^{2}$ 연세대학교 의과대학 세브란스병원 안과학교실 시기능개발연구소 \\ ${ }^{1}$ Department of Ophthalmology, Yonsei University Wonju College of Medicine, Wonju, Korea \\ ${ }^{2}$ Institute of Vision Research, Department of Ophthalmology, Yonsei University College of Medicine, Seoul, Korea
}

Purpose: To describe the effects of ciliary body tumor posterior extension on clinical characteristics and surgical outcomes.

Methods: A chart review was performed on patients with ciliary body tumor that visited between January 1, 2005 and December 31, 2015. Patients were divided into three groups based on tumor posterior extension, as follows; group 1, posterior extension within $6 \mathrm{~mm}$ of the limbus; group 2, posterior extension of 6-12 $\mathrm{mm}$; and group 3, posterior extension of more than $12 \mathrm{~mm}$ from the limbus.

Results: Sixty-one patients were screened, and 49 had pathologically confirmed ciliary body tumors. Malignant melanoma (28 cases, 57.1\%) was the most common type of ciliary body tumor. Benign neoplasm was common when the tumor was confined to the ciliary body (group 1), and a higher malignancy rate was observed in those with advanced posterior extension (groups 2,3 ) than in group 1 $(p=0.001)$. Final visual acuity was poorer in patients with advanced posterior extension $(p<0.001)$, and postoperative retinal detachment rates were higher in these patients ( $p=0.007$ ).

Conclusions: Ciliary body tumors with advanced posterior extension had a higher malignancy rate and were associated with poorer visual outcomes.

Keywords: Ciliary body; Malignant melanoma; Lamellar sclera uvectomy; Posterior extension

Ciliary body tumors can take the form of adenoma, malignant melanoma, and other metastatic tumors [1-3]. Unlike other anterior segment tumors, ciliary body tumors can become relatively large because they are covered by the iris [2]. Treatment includes observation, surgical removal with lamellar sclerouvectomy (LSU), full-thickness eyewall resection, radiation therapy with brachytherapy, proton ir- radiation, and gamma knife surgery [3-6]. In patients with large ciliary body tumor, eyes with neovascular glaucoma, or in which other treatments have failed, enucleation can be performed [7]. Treatment selection depends on tumor size, character, location, presence of symptoms and clinical circumstances $[4,7]$.

Previous studies have tended to view ciliary body tumor
Address reprint requests to Sung Chul Lee, MD, PhD

Institute of Vision Research, Department of Ophthalmology, Yonsei University College of Medicine, \#50 Yonsei-ro, Seodaemun-gu, Seoul 03722, Korea

Tel: 82-2-2228-3570, Fax: 82-2-312-054

E-mail:SUNGLEE@yuhs.ac
Received: 2018. 9. 12

Revised: 2018. 10.5

Accepted: 2018. 10. 22 
as a part of choroidal tumor and concluded that the surgical and pathological outcomes of ciliary body tumors depend on tumor size [8-10]. Furthermore, choroidal melanoma is rare in Asians, and hence, reports on the topic are limited [1,11].

Because local invasion is a characteristic of malignant tumors, tumor extension over the ciliary body is probably associated with pathological differences [12]. Additionally, the resection of large amounts of tissue is associated with poorer visual outcomes. In the present report, we describe differences between demographic factors, initial presentations, tumor pathologies, surgeries performed, and surgical outcomes of ciliary body tumors with respect to tumor posterior extension.

\section{Materials and Methods}

A retrospective chart review was performed on patients with a ciliary body tumor that visited between January 1, 2005 and December 31, 2015. The Institutional Review Board of Yonsei University College of Medicine approved this study, which also complied with the tenets of the Declaration of Helsinki.

A ciliary body tumor was defined as a tumor confined to the ciliary body or a tumor involving both choroid and ciliary body with maximum height at the ciliary body. Primary ciliary body tumors and inflammatory lesions were included. Twenty-seven cases of ciliary body melanoma were described in our previous report, and these patients were included in the present study [1]. Patients with a history of surgical or radiologic treatment for a ciliary body tumor before visiting our institution were excluded, as were patients with a known metastatic tumor since treatments like chemotherapy could have affected ciliary body tumors. All patients underwent a thorough ophthalmic evaluation, which included ultrasound biomicroscopy (UBM). When malignancy was suggested, brain and orbital magnetic resonance imaging (MRI) with abdominal computed tomography or whole-body positron emission tomography were performed. Visual acuity was substituted to logarithm of minimal angle of resolution value for statistical analysis. Anterior chamber reaction was defined when there was an inflammatory cell present at the anterior chamber. The shallow anterior chamber was defined when peripheral anterior chamber depth was less than 1/4 of corneal thickness. Cataract was defined when localized cataract occurs near the lesion or when it is sufficiently severe for surgical removal.
The vitreous cell was detected with the slit-lamp biomicroscopy or 90D lens.

Tumor heights, largest basal diameters, and posterior extensions from limbus and numbers of clock hours involved were determined by MRI or UBM. Patients were divided into groups depending on posterior extensions from limbus: group , within $6 \mathrm{~mm}$ of the limbus (i.e., tumor confined to the limbus); group 2, 6-12 $\mathrm{mm}$ from the limbus (i.e., tumor extension over the ora serrata but not over the equator); and group 3, posterior extension $>12 \mathrm{~mm}$ (i.e., tumor extension beyond the equator). The 6th edition of the Tumor, Node, Metastasis (TNM) classification for malignant ciliary body tumor and choroidal melanoma was used for TNM staging [13].

Patients were warned about possible malignancy, and surgery or biopsy was recommended when malignancy was suspected. However, patients with a smooth surfaced tumor confined to the ciliary body were informed that observation was an option, and that surgery would only be necessary if the tumor progressed. Patients were also informed that enucleation and radiotherapy were treatment options. All patients provided written informed consent.

Surgery was performed by a single surgeon (S.C.L.) using previously described methods [14]. In brief, LSU was performed with a $2.0 \mathrm{~mm}$ safety margin leaving $80-90 \%$ of scleral thickness. A scleral flap hinge was placed anteriorly for small tumors (group 1), but when tumors extended over the ora serrata, the hinge was placed posteriorly. For large tumors, when posterior margin of tumor was difficult to approach, limited pars plana vitrectomy (PPV) was used to decompress the orbit, a careful incision was then made to inner scleral fibers to expose the uvea, and removal was performed with inner scleral fibers and normal surrounding uveal tissue. The scleral flap was then sutured back to its original position. After scleral suturing was complete, the eyeball was inflated with balanced salt solution, and eyes were inspected with a microscope and indirect ophthalmoscope to detect possible complications. When retinal detachment was present or vitreous had been lost during the operation, total PPV (TPPV) was performed; a silicone oil tamponade was used when needed. In patients with cataract or lens damage, lensectomy was also performed. After October 2006, Ru-106 plaque brachytherapy became available at our facility. During operations, a frozen section biopsy of the tumor was performed, and in patients with a malignant tumor, brachytherapy was combined with LSU. In patients that underwent biopsy of the ciliary body tu- 
mor, those with benign tumors were not treated further, but if malignancy was found or inconclusive results were obtained, additional procedures were performed.

The Kruskal-Wallis test, one-way analysis of variance, the chi-square test, and Fisher's exact test were used to compare group characteristics and outcomes. Pearson correlation coefficient was used to compare relationship between tumor extensions and visual acuity. The Kaplan-Meier method was used to compare group distant metastasis rates and survivals. Linear regression and receiver operating characteristic curves were used to compare outcomes with respect to posterior extension and TNM stage. Results were analyzed using SPSS version 21.0 (IBM Corp., Armonk, NY, USA), and statistical significance was accepted for $p<0.05$.

\section{Results}

Initial findings were evaluated in 61 patients (61 eyes), and surgical outcomes and long-term findings were evaluated in 56 (5 patients were lost to follow-up). Forty-nine patients had a pathologically confirmed ciliary body tumor. Patients were assigned to three groups based on extent of posterior tumor extension, as follows: 25 were assigned to group 1, in which tumors were confined to the ciliary body (posterior extension within $6 \mathrm{~mm}$ of the limbus); 20 were assigned to group 2, in which tumors extended over the ora serrata but not over the equator (posterior extension of 6-12 $\mathrm{mm}$ ); and 16 were assigned to group 3, in which tumors extended beyond the equator. Thus 36 patients had a ciliary body tumor with advanced posterior extension (posterior choroidal extension of $\geq 6 \mathrm{~mm}$ of the limbus). The average follow-up period was 44 months, and group follow-up periods were similar (Table 1).

No significant intergroup differences were observed for age, sex, or involved eye. In group 1, 1 patient had atopic dermatitis and 1 had Behçet's disease. In group 2, 1 patient underwent total thyroidectomy for thyroid cancer, which was not metastatic. Although past history of tuberculosis was different between groups, no patient had tuberculoma.

Initial findings are presented in Table 2. Tumor base (mm) were significantly different in the three groups. More clock hours were involved in the group with advanced posterior extension (groups 2 and 3) than in group 1, but no significant difference was observed between groups 2 and 3. Vitreous opacity was present in 2 patients, 1 patient in each group 2 and 3. Grades of vitreous opacity were grade $2+$ in group 2 and grade $1+$ in group 3 . Retinal detachment was only encountered in patients with choroidal involvement $(p=0.003)$. Retinal detachment without macular involvement occurred in 5 patients $(25.0 \%)$ in group 2 , and in $4(25.0 \%)$ in group 3. Retinal detachment with macular involvement was seen in 2 patients $(3.3 \%)$ (1 patient in each of groups $2[5.0 \%]$ and $3[6.3 \%]$ ).

Primary treatments are detailed in Table 3 . In group 1, 6 patients were treated by observation alone; average tumor size was $2.17 \times 1.11 \mathrm{~mm}$ and all were smooth surfaced. These patients underwent 42.0 months (3-107 months) of observation

Table 1. Demographics of the three ciliary body tumor groups

\begin{tabular}{|c|c|c|c|c|c|}
\hline & Group 1 & Group 2 & Group 3 & Total & $p$-value \\
\hline Number of Pts & 25 & 20 & 16 & 61 & \\
\hline Age & $46.64 \pm 13.81$ & $49.20 \pm 16.00$ & $53.06 \pm 13.35$ & $49.16 \pm 14.44$ & $0.258^{*}$ \\
\hline Sex (male) & $7(28.0)$ & $8(40.0)$ & $8(50.0)$ & $23(37.7)$ & $0.333^{\dagger}$ \\
\hline Involved eye & & & & & $0.805^{\dagger}$ \\
\hline OD & $10(40.0)$ & $10(50.0)$ & $8(50.0)$ & $28(45.9)$ & \\
\hline OS & $15(60.0)$ & $10(50.0)$ & $8(50.0)$ & $33(54.1)$ & \\
\hline Tuberculosis Hx & $0(0.0)$ & $4(20.0)$ & $2(12.5)$ & $6(9.8)$ & $0.046^{\ddagger}$ \\
\hline Malignancy $\mathrm{Hx}$ & $0(0.0)$ & $1(5.0)^{\S}$ & $0(0.0)$ & $1(1.6)$ & $0.590^{\ddagger}$ \\
\hline Mean F/U (months) & $48.73 \pm 33.86$ & $42.74 \pm 30.78$ & $40.40 \pm 33.11$ & $44.46 \pm 32.25$ & $0.720^{\sqcap}$ \\
\hline
\end{tabular}

Values are presented as mean \pm standard deviation or number (\%).

Pts = patients; $\mathrm{OD}=$ oculus dexter; $\mathrm{OS}=$ oculus sinister; $\mathrm{Hx}=$ history; $\mathrm{F} / \mathrm{U}=$ follow up.

*one patient had previously undergone total thyroidectomy for thyroid cancer; ${ }^{\dagger}$ Kruskal-Wallis test; ${ }^{\ddagger}$ chi-square test; ${ }^{\circledR}$ Fisher's exact test;

пone-way analysis of variance. 
Table 2. Initial presentations in the three study groups

\begin{tabular}{|c|c|c|c|c|c|}
\hline & Group 1 (25) & Group $2(20)$ & Group 3 (16) & Total & $p$-value \\
\hline Initial BCVA (LogMAR) & $0.31 \pm 0.48$ & $0.63 \pm 0.57$ & $0.64 \pm 0.79$ & $0.50 \pm 0.62$ & $0.008^{*}$ \\
\hline Initial BCVA over 0.5 (decimal) & $19(76.0)$ & $9(45.0)$ & $7(43.8)$ & $35(57.4)$ & $0.060^{+}$ \\
\hline Tumor base (mm) & $3.69 \pm 1.72$ & $9.61 \pm 1.77$ & $13.43 \pm 1.50$ & $8.42 \pm 4.34$ & $<0.001^{*}$ \\
\hline Tumor extension form limbus (mm) & $2.03 \pm 1.92$ & $9.29 \pm 1.73$ & $15.61 \pm 2.54$ & $8.28 \pm 5.88$ & $<0.001^{*}$ \\
\hline Tumor height (mm) & $3.06 \pm 2.13$ & $9.24 \pm 3.30$ & $8.93 \pm 2.25$ & $6.87 \pm 3.91$ & $<0.001^{*}$ \\
\hline Involved clock hours & $1.33 \pm 0.51$ & $2.08 \pm 0.71$ & $2.47 \pm 6.18$ & $1.89 \pm 0.77$ & $<0.001^{*}$ \\
\hline Shallow anterior chamber & $2(8.0)$ & $0(0.0)$ & $0(0.0)$ & $2(3.3)$ & $0.333^{\ddagger}$ \\
\hline Anterior chamber reaction & $7(28.0)$ & $2(10.0)$ & $0(0.0)$ & $9(14.8)$ & $0.035^{\ddagger}$ \\
\hline Cataract & $7(28.0)$ & $2(10.0)$ & $3(18.8)$ & $12(19.7)$ & $0.366^{\ddagger}$ \\
\hline Vitreous cell & $2(8.0)$ & $9(45.0)$ & $7(43.8)$ & $18(29.5)$ & $0.007^{\ddagger}$ \\
\hline Vitreous opacity & $0(0.0)$ & $1(5.0)$ & $1(6.3)$ & $2(3.3)$ & $0.508^{\ddagger}$ \\
\hline Macular edema & $1(4.0)$ & $0(0.0)$ & $0(0.0)$ & $1(1.6)$ & $1.000^{\ddagger}$ \\
\hline Retinal detachment & $0(0.0)$ & $6(30.0)$ & $5(31.3)$ & $11(18.0)$ & $0.003^{\ddagger}$ \\
\hline
\end{tabular}

Values are presented as mean \pm standard deviation or number (\%).

$\mathrm{BCVA}=$ best-corrected visual acuity; LogMAR = logarithm of minimal angle of resolution.

${ }^{*}$ Kruskal-Wallis test; ${ }^{\dagger}$ Chi-square test; ${ }^{\dagger}$ Fisher's exact test.

Table 3. Primary treatments in the three study groups

\begin{tabular}{|c|c|c|c|c|}
\hline & Group 1 (25) & Group 2 (20) & Group 3 (16) & Total \\
\hline Observation & $6(24.0)$ & $0(0.0)$ & $0(0.0)$ & $6(9.8)$ \\
\hline Biopsy & $1(4.0)$ & $3(15.0)$ & $2(12.5)$ & $6(9.8)$ \\
\hline LSU +/- Brachy & $8(32.0)$ & $1(5.0)$ & $0(0.0)$ & $9(14.8)$ \\
\hline LSU + Cat +/- Brachy & $1(4.0)$ & $1(5.0)$ & $0(0.0)$ & $2(3.3)$ \\
\hline LSU + TPPV +/- Brachy & $4(16.0)$ & $10(50.0)$ & $3(18.8)+1^{*}$ & $17(27.9)+1^{*}$ \\
\hline LSU + TPPV + Cat +/- Brachy & $2(8.0)$ & $3(15.0)+2^{*}$ & $8(50.0)$ & $13(21.3)+2^{*}$ \\
\hline Combined silicone oil injection & $0(0.0)$ & $8(40.0)$ & $10(62.5)$ & $18(29.5)$ \\
\hline External radiation & $0(0.0)$ & $1(5.0)$ & $1(6.3)$ & $2(3.3)$ \\
\hline Enucleation & $0(0.0)$ & $0(0.0)$ & $1(6.3)$ & $1(1.6)$ \\
\hline Follow-up loss & $3(12.0)$ & $1(5.0)$ & $1(6.3)$ & $5(8.2)$ \\
\hline
\end{tabular}

Values are presented as number (\%).

LSU = lamellar sclerouvectomy; Brachy = brachy therapy; Cat = cataract extraction; TPPV = total pars plana vitrectomy.

*Patients with malignancy or inconclusive biopsy results.

with no specific progression and remain under observation without specific treatment. Six patients underwent biopsy, in group 1 biopsy revealed adenoma in one patient. In group 2, biopsy revealed 1 melanocytic nevus, 1 malignant melanoma, and 1 was inconclusive. The patient with inconclusive findings underwent LSU + TPPV + lensectomy, and schwannoma was confirmed. One patient in group 3 had mesectodermal leiomyo$\mathrm{ma}$, and the other with inconclusive findings confirmed to have malignant melanoma by LSU + TPPV. Patients with advanced posterior extensions required more complex surgery. In group 1 , LSU with or without brachy therapy was most commonly performed surgery ( 8 patients, $32 \%$ ). In group 2 , patients also required TPPV with LSU with or without brachy therapy (10 patients, 50\%). In group 3, cataract extraction was combined with TPPV and LSU with or without brachy therapy (8 patients, $50 \%)$.

Brachytherapy was used in combined treatments in 21 (75.0\%) of 28 patients with malignant melanoma and in 3 of the 28 
Table 4. Pathologies in the three study groups who have confirmed by pathologic examination

\begin{tabular}{|c|c|c|c|c|c|}
\hline & Group 1 (16) & Group 2 (18) & Group 3 (15) & Total (49) & $p$-value \\
\hline Malignant & $5(31.3)$ & $10(55.6)$ & $14(93.3)$ & $29(59.2)$ & $0.001^{*}$ \\
\hline Malignant melanoma & $5(31.3)$ & $9(50.0)$ & $14(93.3)$ & $28(57.1)$ & $0.001^{*}$ \\
\hline Epithelioid & $2(12.5)$ & $3(16.7)$ & $4(26.7)$ & $9(18.4)$ & $0.086^{\dagger}$ \\
\hline Mixed & $1(6.3)$ & $0(0.0)$ & $3(20.0)$ & $6(12.2)$ & \\
\hline Spindle & $0(0.0)$ & $4(22.2)$ & $2(13.3)$ & $5(10.2)$ & \\
\hline Squamous cell carcinoma & $0(0.0)$ & $1(5.6)$ & $0(0.0)$ & $1(2.0)$ & $1.000^{\dagger}$ \\
\hline Benign & $11(68.8)$ & $8(44.4)$ & $1(6.7)$ & $20(40.8)$ & \\
\hline Adenoma & $4(25.0)$ & $2(11.1)$ & $0(0.0)$ & $6(12.2)$ & $0.117^{\dagger}$ \\
\hline Leiomyoma & $1(6.3)$ & $4(22.2)$ & $1(6.7)$ & $6(12.2)$ & $0.342^{\dagger}$ \\
\hline Nevus & $1(6.3)$ & $0(0.0)$ & $0(0.0)$ & $1(2.0)$ & $0.633^{\dagger}$ \\
\hline Melanocytoma & $3(18.8)$ & $0(0.0)$ & $0(0.0)$ & $3(6.1)$ & $0.055^{\dagger}$ \\
\hline Schwannoma & $0(0.0)$ & $1(5.6)$ & $0(0.0)$ & $1(2.0)$ & $1.000^{\dagger}$ \\
\hline Neurogenic tumor & $0(0.0)$ & $1(5.6)$ & $0(0.0)$ & $1(2.0)$ & $1.000^{\dagger}$ \\
\hline Non-teratoid medulloepithelioma & $1(6.3)$ & $0(0.0)$ & $0(0.0)$ & $1(2.0)$ & $0.633^{\dagger}$ \\
\hline Fibrosis with pigment & $1(6.3)$ & $0(0.0)$ & $0(0.0)$ & $1(2.0)$ & $0.633^{\dagger}$ \\
\hline
\end{tabular}

Values are presented as number (\%).

${ }^{*}$ Chi-square test; ${ }^{\dagger}$ Fisher's exact test.

that developed local recurrence; 4 of 28 patients that received brachytherapy developed distant metastasis. In 7 patients that did not receive brachytherapy no local recurrence occurred, but 3 developed distant metastasis. Local recurrence and distant metastasis were not associated with brachytherapy (local recurrence: $p=$ 0.551 , distant metastasis: $p=0.318)$. One patient in group 3 with a $14.86 \times 10.08 \mathrm{~mm}$ sized tumor with scleral pigmentation above the tumor underwent primary enucleation as symptoms were suggestive of extrascleral extension. However, pathology findings indicated the absence of extrascleral extension.

Ciliary body tumor was pathologically confirmed in 49 cases (Table 4). A significantly higher rate of malignancy was observed in the advanced posterior extension group (groups 2 and 3) than in group 1. Malignant melanoma rates were significantly different between all three groups $(p<0.001)$. Cell type of melanoma was reported in 20 patients, but no significantly intergroup difference was found in terms of cell composition. Squamous carcinoma was present in 1 patient in group 2, and additional tests determined it originated from lung cancer. No significant intergroup difference was observed between pathologic diagnoses, expect for malignant melanoma.

Surgical outcomes and long-term follow-up results are presented in Table 5. Although, best-corrected visual acuity (BCVA) values at initial visit were not significantly different in the groups, patients with advanced posterior extension had significantly poorer final BCVA values $(p<0.001)$. Posterior extension of tumor from the limbus was more strongly associated than largest basal diameters with final BCVA values and changes in BCVA values (Fig. 1). Linear regression was used to compare the associations between posterior extension and TNM staging and final visual acuity. Although both TNM staging and posterior extension were found to be significantly related to final visual acuity ( $p$ $<0.001$ ), posterior extension classification showed a stronger correlation with final BCVA (posterior extension $\mathrm{R}^{2}=0.433$, TNM staging $\mathrm{R}^{2}=0.366$ ).

Tumors extending beyond the ora serrata (groups 2 and 3) were found to be significantly more prone to retinal detachment ( $p$ $=0.007)$. One patient in each group underwent secondary enucleation due to local tumor recurrence. In group 2, 1 patient underwent enucleation for a residual tumor, and 1 patient underwent enucleation for sympathetic ophthalmia; in group 3, there was 1 case of secondary glaucoma with pain, and 2 cases of iris neovascularization. Secondary enucleation was performed within 27 months (0-90 months) on average after first operation. Time differences from primary operation to enucleation were similar in the three groups (group 1: 41 months, group 2: 15 months, group 3: 35.5 months, $p=0.638$ ). The total number of operations required differed significantly in the three groups, and patients 
Table 5. Outcomes in the three study groups (patients placed under observation are included)

\begin{tabular}{|c|c|c|c|c|c|}
\hline & Group 1 (22) & Group 2 (9) & Group 3 (15) & Total (56) & $p$-value \\
\hline Final BCVA (LogMAR) & $0.77 \pm 1.26$ & $2.37 \pm 1.85$ & $3.73 \pm 1.23$ & $2.09 \pm 1.87$ & $<0.001^{*}$ \\
\hline Final BCVA over 0.5 (decimal) & $12(54.5)$ & $3(15.8)$ & $0(0.0)$ & $15(26.8)$ & $<0.001^{\dagger}$ \\
\hline Final BCVA over 0.1 (decimal) & $18(81.8)$ & $6(31.6)$ & $0(0.0)$ & $24(42.9)$ & $<0.001^{\dagger}$ \\
\hline BCVA change (LogMAR) & $-0.45 \pm 1.17$ & $-1.73 \pm 1.70$ & $-3.08 \pm 1.51$ & $-1.59 \pm 1.78$ & $<0.001^{*}$ \\
\hline Improved visual acuity & $6(27.3)$ & $1(5.3)$ & $0(0.0)$ & $7(12.5)$ & $0.039^{+}$ \\
\hline Number of total operations & $1.00 \pm 0.82$ & $1.68 \pm 0.82$ & $2.20 \pm 1.20$ & $1.55 \pm 1.043$ & $0.005^{*}$ \\
\hline Retinal detachment & $0(0.0)$ & $5(26.3)$ & $5(33.3)$ & $10(17.9)$ & $0.007^{\dagger}$ \\
\hline Vitreous hemorrhage & $0(0.0)$ & $3(15.8)$ & $2(13.3)$ & $5(8.9)$ & $0.110^{\dagger}$ \\
\hline Glaucoma & $2(9.1)$ & $2(10.5)$ & $3(20.0)$ & $7(12.5)$ & $0.602^{\dagger}$ \\
\hline Neovascularization of the iris & $0(0.0)$ & $2(10.5)$ & $2(13.3)$ & $4(7.1)$ & $0.173^{\dagger}$ \\
\hline Cataract & $3(13.6)$ & $2(10.5)$ & $3(20.0)$ & $8(14.3)$ & $0.797^{\dagger}$ \\
\hline Phthisis & $0(0.0)$ & $3(15.8)$ & $0(0.0)$ & $3(5.4)$ & $0.051^{\dagger}$ \\
\hline Enucleation $^{\ddagger}$ & $1(4.5)$ & $3(15.8)$ & $4(26.7)$ & $8(14.3)$ & $0.186^{\dagger}$ \\
\hline
\end{tabular}

Values are presented as mean \pm standard deviation or number (\%).

$\mathrm{BCVA}=$ best-corrected visual acuity; LogMAR = the logarithm of minimal angle of resolution.

${ }^{*}$ Kruskal-Wallis test; ${ }^{\dagger}$ Fisher's exact test; ${ }^{\ddagger}$ primary enucleated patient was excluded.

A

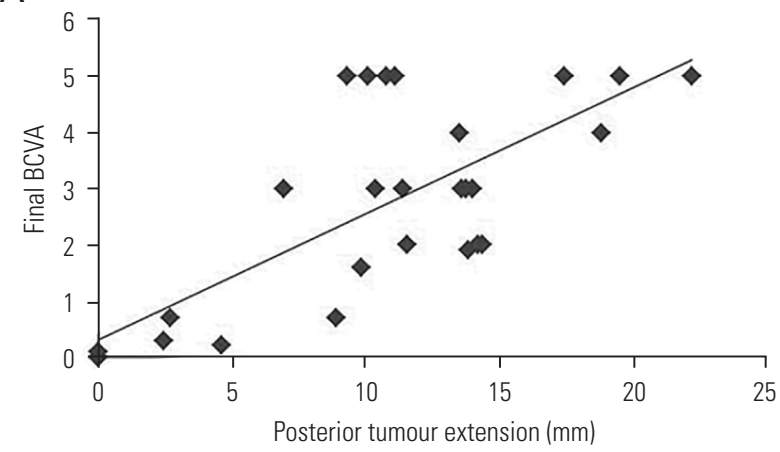

C

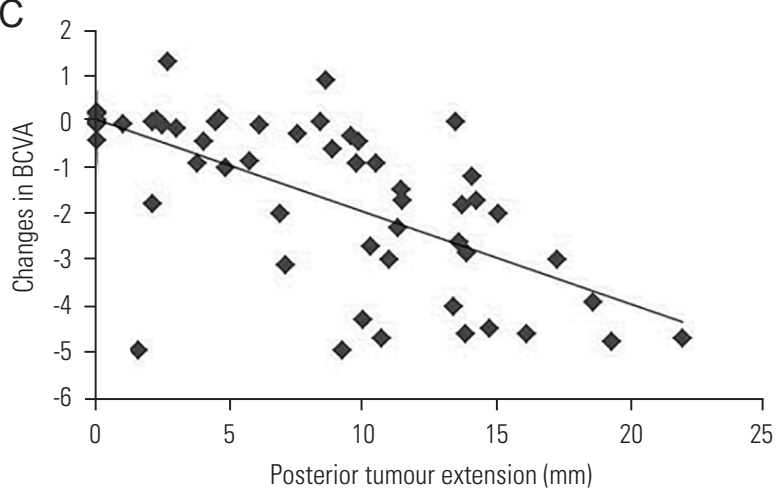

B
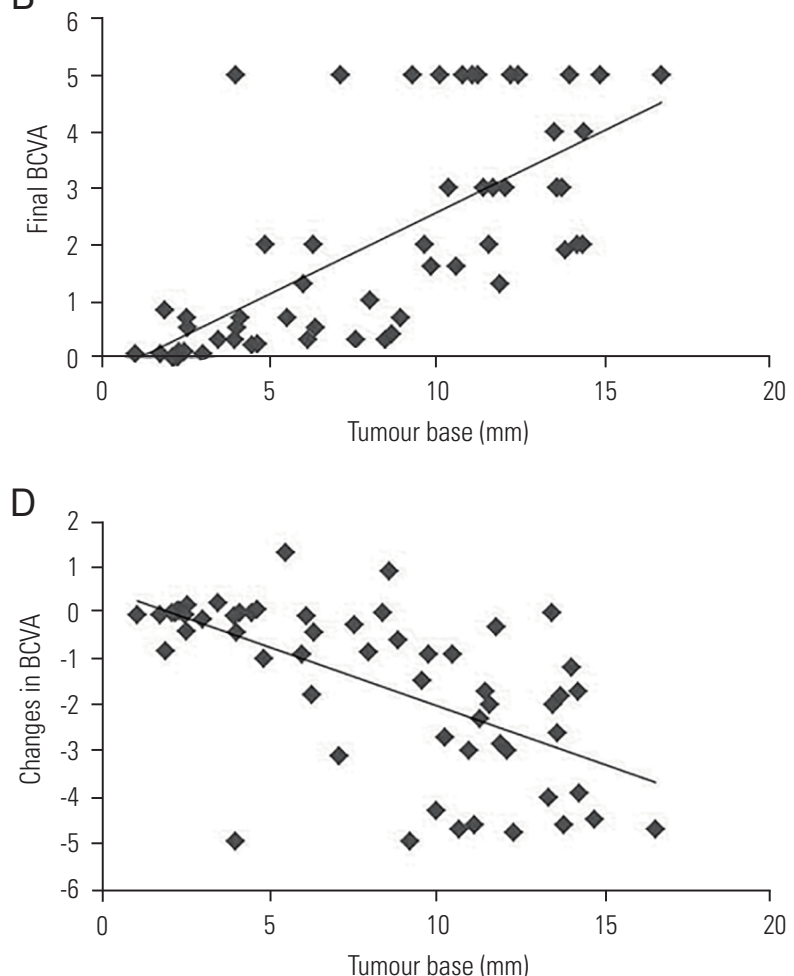

Figure 1. Correlation between posterior tumor extension from the limbus and largest tumor base with final and changes in best-corrected visual acuity (BCVA), in logarithm of minimal angle of resolution scale. Coefficients of determination ( $R^{2}$ ) were (A) 0.479 , (B) 0.467 , (C) 0.415 , and (D) 0.376 . 
Table 6. Outcomes of ciliary body melanoma in the three study groups

\begin{tabular}{lccccc}
\hline & Group 1 (5) & Group 2 (9) & Group 3 (14) & Total (28) & $p$-value \\
\hline Residual tumor & $0(0.0)$ & $1(11.1)$ & $0(0.0)$ & $1(3.6)$ & 0.500 \\
Local recurrence & $1(20.0)$ & $1(11.1)$ & $1(7.1)$ & $3(10.7)$ & 0.750 \\
Distant metastasis & $1(20.0)$ & $2(22.2)$ & $4(28.6)$ & $7(25.0)$ & 1.000 \\
Mortality & $0(0.0)$ & $2(22.2)$ & $4(28.6)$ & $6(21.4)$ & 0.271 \\
\hline
\end{tabular}

Values are presented as $n$ (\%).

*Fisher's exact test.

with advanced posterior extension (groups 2 and 3) required more operations $(p=0.001)$.

Surgical outcome of 28 patients with malignant melanoma were evaluated for local recurrence and distant metastasis, and tumor-related mortality (Table 6). Local recurrence was not significantly different in the three groups $(p=0.750)$. On average, local recurrence occurred within 25 months after primary operation (37 months in group 1, 29 months in group 2, and 8 months in group 3), and distant metastasis was encountered within 38 months (range 7-88 months) (87 months in group 1, 29 months [7-50 months] in group 2, and 30 months [22-38 months] in group 3). No significantly intergroup difference was observed in terms of the occurrence of distant metastasis ( $p=$ $1.000)$ or time to metastasis $(p=0.457)$. Six patients $(21.4 \%)$ experienced tumor-related mortality. All 6 patients in groups 2 and 3 with distant metastasis expired. These patients had an average age of 56 years (range 36-69 years) at time of death, which occurred at an average of 51.8 months (21-61 months) after initial visits. In group 2, patients had an average age of 54.5 years (53-56 years), and underwent 39 months (21-57 months) of observation; in group 3, patients had an average age of 56.75 years (36-69 years), and underwent 43.25 months (26-61 months) of observation. Melanoma cell type was not significantly related to local recurrence $(p=0.084)$ nor distant metastasis ( $p=0.672$ ). The Kaplan-Meier analysis was used to compare groups with respect to distant metastasis and patient survival, but no intergroup difference was found (metastasis $p$ $=0.901$, and survival $p=0.560$ ).

\section{Discussion}

In the present study, age, sex, involved eye, and medical history were not found to be related to extents of posterior extension of ciliary body tumors. Vitreous cell (29.5\%), cat- aract (19.7\%) and anterior chamber reaction (14.8\%) were the most common initial presentations of ciliary body tumors. Furthermore, even for small tumors confined to the ciliary body, $50 \%$ of patients exhibited either anterior chamber reaction or cataract development. Checking for possible ciliary body tumor in cases of unexplained vitreous cell, cataract, or anterior chamber reaction could be beneficial in terms of reaching an early diagnosis.

Among the 49 pathologically confirmed cases of ciliary body tumors, the most common etiology was malignant melanoma (28 patients, 57.1\%). When a ciliary body tumor was confined within the ciliary body, benign causes, such as, adenoma and melanocytoma, were common. Tumors with posterior extension over the ora serrata were more common among malignant cases. However, although $68.8 \%$ of tumors confined to the ciliary body were benign, $31.3 \%$ were malignant melanomas. Thus, regardless of how small a tumor is, patients should be warned about the possible of malignancy before pathologic confirmation. Additionally, 40.8\% of ciliary body tumors with posterior extension over the equator were benign, and thus, primary enucleation should be considered with caution.

Malignant melanomas appear to occur less frequently in Koreans than in Caucasians. Naumann and Rummelt [15] reported $69.1 \%$ of anterior segment tumors were malignant melanoma, and Shields et al. [3] reported $85 \%$ of uveal and ciliary body tumors removed by LSU were malignant melanomas. In the present study, only $57 \%$ of 49 had malignant melanoma. This lower ratio of malignant melanoma for ciliary body tumors could be due to a lower incidence of melanoma in Asian populations. However, the rate of epithelioid type melanoma was high in the present study, and of 20 patients with ciliary body melanoma, 9 patients had epithelioid type melanoma and only 5 patients had spindle type melanoma. Although, histologic reports on ciliary body 
tumor alone is scarce, Naumann and Rummelt [15] reported $17.0 \%$ epithelioid and $23 \%$ spindle cell types in anterior segment tumors, and Shammas and Blodi [10] reported 46.1\% spindle and $53.9 \%$ of tumors were of the mixed or epithelioid types in choroidal and uveal melanoma cases. Sakamoto et al. [16], discussed differences between the histologic findings of uveal melanoma in Japanese patients, and found that epithelioid cell melanoma more common in Japanese (17\%) than Hispanic and Caucasian patients (6\%) [17].

LSU for a ciliary body tumor does not increase the risk of metastasis and is relatively safe $[3,18,19]$. However, complications, such as, cataract, hemorrhage, hyphema, glaucoma, retinal detachment, and residual tumor, can occur $[1,14,18,20]$. Furthermore, the complications of LSU increase with choroidal involvement by ciliary body tumors $[1,3]$. Vitreous hemorrhage is the most common complication of LSU, with reported rates of 9-83\% $[1,14,18,20]$. This extremely wide range is probably due to different definitions of vitreous hemorrhage. In the present study, 5 patients $(8.9 \%)$ developed vitreous hemorrhage that either required surgery or constantly affected visual acuity, and all patients with vitreous hemorrhage had tumor posterior extension over the ora serrata, but this difference was not significant between groups. In fact, retinal detachment was the only complication that significantly different between our three study groups. Retinal detachment developed in 10 patients (17.9\%), and all cases had choroidal involvement.

Residual tumor after LSU is of concern, as it has a reported local recurrence rate of $0-41 \%[18,19,21]$. Cryotherapy and adjunctive brachytherapy are still viable options for reducing residual tumor and local recurrences [22]. After treatment with cryotherapy and adjunctive brachytherapy, only 3 of our patients $(10.7 \%)$ developed local recurrence. One patient in group 2 with a $7.12 \times 10.06 \mathrm{~mm}$ tumor underwent LSU + $\mathrm{TPPV}+$ lensectomy and internal choroidectomy, but total excision was impossible due to intractable vitreous hemorrhage. Enucleation was performed 3 weeks after the primary operation and 5 months later liver metastasis developed. Thus, total tumor resection is important, and adjunctive methods, such as, cryotherapy or brachytherapy, are needed because complete tumor removal is difficult to verify during the surgical process. We managed to save $47(83.9 \%)$ of 56 eyes, although some patients had a ciliary body tumor extending over the equator. Forty-four patients underwent LSU as the primary operation or after biopsy, and 37 (84.1\%) of eyes were salvaged. This result is comparable to those of previous studies with reported secondary enucleation rates of $16-29 \%[1,3,23]$.

Risk factors of distant metastasis include choroidal melanoma, age, largest basal diameters, ciliary body involvement, and local recurrence [24,25]. In the present study, 7 patients $(25.0 \%)$ developed distant metastasis, but no significant difference in age, largest basal diameters, tumor height, or number of involved clock hours was observed between patients with or without metastasis. LSU + TPPV + lensectomy + brachytherapy was performed as the primary operation in 4 patients; 1 patient (group 2) underwent biopsy and then LSU + TPPV + lensectomy, and 1 patient (group 3) underwent gamma knife surgery as a primary treatment. All 7 patients developed liver metastasis. Six patients (21.4\%) experienced cancer-related mortality. Only 1 patient in group 1 is currently under observation.

Regarding final visual acuity, Shields et al. [3] reported that $26 \%$ of patients who received LSU form ciliary body and choroidal tumor had a visual acuity of more than 20/30 after LSU, but they did not mention tumor size or extension. Five patients (33.3\%) in group 1 that underwent LSU had a final BCVA of $>0.7$, whereas no patient achieved this in group 2 or 3 , presumably because patients with an advanced posterior extension underwent complex operations and had high rates of complications. Additionally, posterior extension of tumor affected final visual acuity more than tumor size, because of differences between extents of retina invasion. Moreover, vitreous loss during surgery and complications, such as, retinal detachment, are more likely to be associated with posterior extension than tumor size.

Our study has several limitations. First, the small sample size and short observation period could have affected statistical outcomes. Second, as this was a single center study of only Korean patients, results may not be generalizable to other ethnicities. Further larger-scale studies with longer observation periods on patients with diverse ethnicities are recommended.

Benign neoplasms were common when tumors were confined to the ciliary body, but patients with choroidal involvement showed a higher rate of malignancy. In the present study, malignant melanoma was most common ciliary body tumor, whereas epithelioid type melanoma is reported more commonly than Caucasians [15]. Visual acuity was poor in patients with tumor posterior extension and a risk of retinal 
detachment was high when posterior extension extended over the ora serrata. All malignant tumors should be monitored for local recurrence and distant metastasis.

\section{Conflicts of Interest}

The authors declare no conflicts of interest relevant to this article.

\section{References}

1. Lee CS, Rim TH, Kwon HJ, et al. Partial lamellar sclerouvectomy of ciliary body tumors in a Korean population. Am J Ophthalmol 2013;156:36-42.e1.

2. Marigo FA, Finger PT. Anterior segment tumors: current concepts and innovations. Surv Ophthalmol 2003;48:569-93.

3. Shields JA, Shields CL, Shah P, Sivalingam V. Partial lamellar sclerouvectomy for ciliary body and choroidal tumors. Ophthalmology 1991;98:971-83.

4. Shields JA, Shields CL. Overview of management of posterior uveal melanoma. In: Sadda SV, ed. Retina, 5th ed. Philadelphia: Saunders, 2013; chap. 143.

5. Finger PT. Plaque radiation therapy for malignant melanoma of the iris and ciliary body. Am J Ophthalmol 2001;132:328-35.

6. Gragoudas ES, Goitein M, Verhey L, et al. Proton beam irradiation. An alternative to enucleation for intraocular melanomas. Ophthalmology 1980;87:571-81.

7. Perry JD, Singh AD, Mehta MP. Enucleation for choroidal melanomas. In: Sadda SV, ed. Retina, 5th ed. Philadelphia: Saunders, 2013; chap. 144.

8. Gragoudas ES, Lane AM, Munzenrider J, et al. Long-term risk of local failure after proton therapy for choroidal/ciliary body melanoma. Trans Am Ophthalmol Soc 2002;100:43-8; discussion 48-9.

9. Mäkitie T, Summanen P, Tarkkanen A, Kivelä T. Microvascular density in predicting survival of patients with choroidal and ciliary body melanoma. Invest Ophthalmol Vis Sci 1999;40:2471-80.

10. Shammas HF, Blodi FC. Prognostic factors in choroidal and ciliary body melanomas. Arch Ophthalmol 1977;95:63-9.

11. Hu DN, Yu GP, McCormick SA, et al. Population-based incidence of uveal melanoma in various races and ethnic groups. Am J Ophthalmol 2005;140:612-7.

12. Liotta LA, Kohn EC. The microenvironment of the tumour-host interface. Nature 2001;411:375-9.

13. Kujala E, Kivelä T. Tumor, node, metastasis classification of malignant ciliary body and choroidal melanoma evaluation of the 6th edition and future directions. Ophthalmology 2005;112:1135-44.

14. Damato BE. Local resection of uveal melanoma. Dev Ophthalmol 2012;49:66-80.

15. Naumann GO, Rummelt V. Block excision of tumors of the anterior uvea. Report on 68 consecutive patients. Ophthalmology 1996;103:2017-27; discussion 2027-8.

16. Sakamoto T, Sakamoto M, Yoshikawa H, et al. Histologic findings and prognosis of uveal malignant melanoma in Japanese patients. Am J Ophthalmol 1996;121:276-83.

17. Hudson HL, Valluri S, Rao NA. Choroidal melanomas in Hispanic patients. Am J Ophthalmol 1994;118:57-62.

18. Robertson DM, Campbell RJ, Weaver DT. Residual intrascleral and intraretinal melanoma: a concern with lamellar sclerouvectomy for uveal melanoma. Am J Ophthalmol 1991;112:590-3.

19. Kurt RA, Gündüz K. Exoresection via partial lamellar sclerouvectomy approach for uveal tumors: a successful performance by a novice surgeon. Clin Ophthalmol 2010;4:59-65.

20. Ramasubramanian A, Shields CL, Kytasty $C$, et al. Resection of intraocular tumors (partial lamellar sclerouvectomy) in the pediatric age group. Ophthalmology 2012;119:2507-13.

21. Chang MY, McCannel TA. Local treatment failure after globe-conserving therapy for choroidal melanoma. Br J Ophthalmol 2013;97:804-11.

22. Damato BE, Paul J, Foulds WS. Risk factors for residual and recurrent uveal melanoma after trans-scleral local resection. $\mathrm{Br} J$ Ophthalmol 1996;80:102-8.

23. Memmen JE, McLean IW. The long-term outcome of patients undergoing iridocyclectomy. Ophthalmology 1990;97:429-32.

24. Shields CL, Furuta M, Thangappan A, et al. Metastasis of uveal melanoma millimeter-by-millimeter in 8033 consecutive eyes. Arch Ophthalmol 2009;127:989-98.

25. Ophthalmic Oncology Task Force. Local recurrence significantly increases the risk of metastatic uveal melanoma. Ophthalmology 2016;123:86-91. 